\title{
Analysing and Implementing the Mobility over MANETS using Random Way Point Model
}

\author{
Amandeep Singh Bhatia \\ Department of CSE \& IT \\ Lovely Professional University
}

\author{
Rupinder Kaur Cheema \\ Department of CSE \& IT \\ Lovely Professional University
}

\begin{abstract}
Wireless networks are increasing in popularity with current advances in technology, The architecture of such networks is not based on a centralized base station but on each node which acts as a router and forwards data packets to other nodes in the network. The technologies have driven into new era with the introduction of ad hoc networks and the concept behind the ad hoc networks is it works without the access points. It has features like adaptive, self organizing and decentralized in nature. Due to these specialized features, it has become a popular technology. So, there has been an inevitable need of a good routing protocol in order to establish the connection between the nodes since the mobile nodes can change their topology frequently. The movement of the mobile node is one of the important characteristics because it can affect the performance of the ad hoc network protocol. This paper has analyzed the mobility of the random waypoint model for different routing protocols in mobile ad-hoc network.
\end{abstract}

\section{Keywords}

MANET, Random Waypoint model, Routing Protocols AODV, DSDV, DSR.

\section{INTRODUCTION}

Wireless systems, both mobile and fixed, have become an indispensable part of communication infrastructure. These applications are ranging from simple wireless, low data rate transmitting sensors to high data rate real-time systems, those used for monitoring large retail outlets or real-time broadcasting of sport events [14]. The existing wireless technology is based on point-to-point technology. An example is GSM system with an architecture that is based on mobile nodes communicating directly with central access points. Sometimes there are networks that cannot rely on the centralized connectivity such as Mobile Ad-Hoc Networks (MANET). MANET is a wireless network having mobile nodes with no fixed infrastructure. This kind of networks is used in areas such as environmental monitoring or in rescue operations.

The presence of both wireless communication and mobility makes an ad hoc network unlike a traditional wired network, and it requires the routing protocols used in an ad hoc network based diverse principles. Since mobile ad hoc networks change their topology frequently and routing in these networks is a challenging task. The main task of routing in a network is to detect and maintain the optimal route to send data packets between source and destination with intermediate nodes. The movements are frequently changing in speed, direction and rate that will be affecting the protocols and system designed to support mobility. The mobility model is designed to describe the movement pattern of mobile user, how their location, velocity and acceleration changes over time. Since mobility pattern may play a significant role in determining the protocol performance, it is desirable for mobility model to emulate the movement pattern of the targeted real life application in a reasonable way. The different selection criterions of mobility model can have a major impact on the selection of a routing scheme. Thus, performance has been influenced. The aim of this paper is to analyze the performance of routing protocols with random waypoint mobility model.

\subsection{Ad-Hoc Routing Protocols}

A number of routing protocols for Ad-hoc networks exist and they are classified as proactive and reactive protocols. This work focuses on DSDV (proactive), AODV and DSR (reactive) protocols.

\subsubsection{Destination-Sequenced Distance-Vector (DSDV):}

DSDV as explained in [17] is a distance vector protocol also known as a proactive protocol and a table-driven routing protocol which is derived from the Bellman-Ford routing mechanisms. It has incorporated modifications to address the poor looping properties and time dependent nature of the interconnection topology describing links between mobile hosts. DSDV requires that each mobile host maintains a routing table which lists all available destinations with the number of hops to these destinations .Thus, each hop is forming a network which is required to advertise its own routing table to its "current" neighbors.

\subsubsection{Ad Hoc On Demand Distance Vector (AODV): \\ AODV as presented in [12] is a reactive or Source-initiated On-demand [18] protocol which requires that all mobile hosts obtain routes as needed with little or no reliance on periodic updates. It has been called as a pure on-demand route acquisition system because when connectivity is required each host becomes aware of its neighbors. This can be done by the use of hello messages and a path discovery process is initiated to locate the destination host.}

\subsubsection{Dynamic Source Routing (DSR):}

DSR [19] is another reactive protocol which operates on an entirely on-demand basis, which allows mobile hosts to dynamically discover a source route across multiple network hops to any destination within the ad hoc network. When each data packet sent, it carries a complete ordered list of nodes including the source node in its header. It helps to remove loops as well as routing information in the intermediate mobile host, through which the packet has been forwarded. With this all nodes involved in the transmission of this packet can cache this routing information for future use. 


\section{REVIEW OF LITERATURE}

Author Tracy Camp, Jeff Boleng and Vanessa Davies [1] explained that most of the performance investigations that use the Random Waypoint Mobility Model. The mobile nodes are initially distributed randomly around the simulation area. Random distribution of mobile nodes is not representative of the manner in which nodes distribute themselves when moving. It is flexible, and it appears to create realistic mobility patterns for the way people might move in. One concern with this model is the straight movement pattern created by the mobile node to the next chosen destination.

Author Muhammad Abdulla, Robert Simon [2] found that the inter-contact time distribution under Random Waypoint and Random Direction models differ from the exponential distribution in different ways for small inter-contact times. It has shown that power-law head and exponential tail of intercontact times that are observed in real-world mobility traces can be produced under certain settings in this extended model.

Author Fan Bai, Narayanan Sadagopan, Ahmed Helmy [3] evaluated the performance of DSR, AODV and DSD using a proposed framework not limited to the Random Waypoint Model stating that the model can only be applicable to some scenarios. Author has observed that the protocol performance may vary drastically across mobility and that performance rankings of protocols may vary with the mobility models used.

Author T. Larsson, N. Hedman, B.Mielczarek, and M. Degermark [4] had compared DSR, DSDV and AODV using the Random Waypoint model. In these scenarios where nodes move randomly and also in three realistic scenarios (Conference, Event Coverage and Disaster scenarios) They observed that in most simulations the reactive protocols performed significantly better than DSDV a proactive protocol.

Author Yogesh Chaba, Yudhvir Singh and Manish Joon [5] analyzed the comparative study of two on-demand routing protocols, AODV and DSR based on normalized routing overhead, Packet Delivery Ratio (PDR), and end-to-end delay while varying the number of sources and pause time has been performed [5]. They observed that DSR performs better in terms of overhead and in terms of PDR when compared with AODV.

Author R. Groenevelt, P. Nain, and G. Koole [6] analyzed the message delay in epidemic routing under the assumption that the inter-contact times are exponentially distributed and also experimentally verify the validity of the assumption under Random Waypoint and Random Direction models.

Author Raj Acharya, Aravindhan Venkateswaran and Natarajan Gautam [7] explained that when the nodes do not exhibit total random motion and predictive clustering scheme which significantly improves the temporal stability of the clusters only when it is compared to a mobility-aware nonpredictive scheme. They have shown that there is a tradeoff between the stability and the size of the clusters.

\section{MOBILITY MODEL}

In the past, the wireless networking community relied on simple models such as random waypoint [16]. However, this model has found to be too simplistic very useful in analysis and simulation. On later, it was widely accepted and used in simulations. Recently the researcher has started to focus on the alternative mobility models with different mobility features. This paper is focusing on the development of parsimonious and accurate models describing the random movement of nodes in the Random-Waypoint Mobility Model in mobile wireless networks.

\subsection{Random Waypoint Mobility Model}

The Random Waypoint Mobility Model used by Johnson [10] includes pause times between changes in direction and/or in speed. A mobile node is staying at one location for a certain period of time in beginning. It then travels towards the newly chosen destination at the same speed. Upon arrival, the mobile node takes another break before starting the process again. According to [8] the Random Waypoint Mobility Model depicts a mobile host as remaining in a location for a certain period of time after which a random point within the simulation space is chosen and it travels to that point with a selected speed. The use of Random Waypoint model is really popular in MANET research $[8,9]$.

\section{METHODOLGY}

The overall goal of this work is to measure analyze the random waypoint model mobility for manet routing protocols using Network Simulator (NS2). Basic methodology consisted of first selecting the most representative parameters for a mobile ad-hoc network, then defining and simulating basic scenario and finally, by varying the selected parameters, simulates and evaluates more scenarios.

The mobility and receiver configuration are deployed in the design of MANETs and the mobility configuration defines the random way point model. Random way point model decides the pause times when any changes in speed and direction of the node occurs. The random way point model is random in nature and the process begins with the pause time of the node for a certain amount of time and when it exceeds the time, it starts with the random simulation in the given area. The $\mathrm{X}$ axis and $\mathrm{Y}$-axis is provided with the minimum and maximum speeds for the node to travel in the area and the mobility configuration sets the attributes necessary for speed and configures the mobility profiles.

The random way point model is used in the design of mobile ad hoc networks to provide the speed and distance in a random manner. The start and pause times are provided and when any mobile node is transmitting any data, it will start with a constant time and the nodes can move in a zigzag position with uniform distribution. The $\mathrm{X}$ and $\mathrm{Y}$ are classified as the distance of the coverage area and speed is assigned in a random fashion [14]. The process includes the transmission of data from a source point with a pause time and start to a destination and this could be a random way with a random speed and the bit rate will be constant throughout the transmission. The update frequency is assigned in this model to update the node position and depends upon the small or high frequencies.

The use of directional and Omni directional antennas helps the mobile nodes to transmit form the location in the BSS. Directional antennas are emerge with so many advantages which include better signal strength for transmission, usage for spatial reuse, usage for wide coverage areas and due to the increase in signal strength and coverage results to the better capacity, reduce in the interference and multipath fading [14]. 
There are some cons in using the directional antenna in ad hoc networks which have been stated as hidden node problems, can't able to hear some signals and difficult to find the neighbor locations.

We have considered AODV, DSDV, DSR routing protocols and evaluation is based on the window size which shows the size of buffer in the receivers and total number of packets received, so it will influence the communication procedure.

\subsection{Simulation Setup}

A detailed simulation model based on ns-2 is used in the evaluation.

\subsubsection{The Traffic and Mobility Models}

Constant bit rate (CBR) traffic sources are used. Source destination pairs are spread randomly over the network. Data packets of 512-byte are used. Number of source destination pairs and the packet sending rate in each pair is varied to change the offered load in the network. Random Waypoint model is use in a rectangular field as mobility model. The field configurations used are: $500 \mathrm{~m}$ x $400 \mathrm{~m}$ field with 10,20 and 40 nodes. Here, each packet starts its journey from a random location to a random destination with a randomly chosen speed (uniformly distributed $20 \mathrm{~m} / \mathrm{s}$ ). The pause time, which affects the relative speeds of the mobiles, is fixed. Simulations are run for 150 simulated seconds. Identical traffic and mobility scenarios are used across protocols to gather fair results.

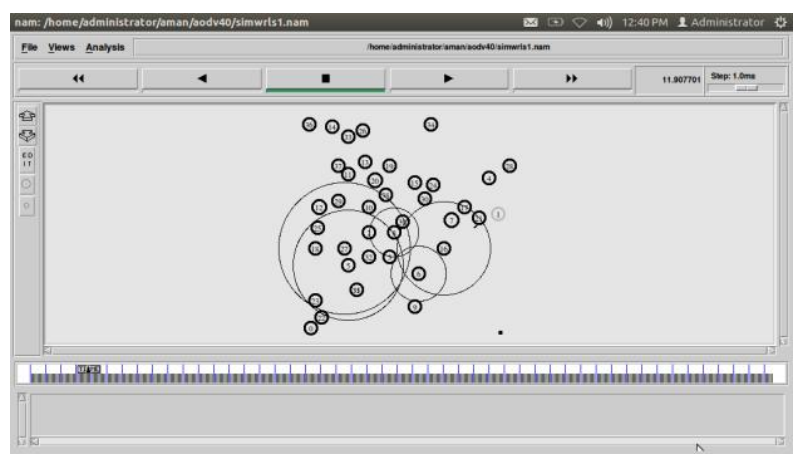

Figure 1: Simulation setup with 40 nodes

\subsubsection{Performance metrics}

4.1.2.1 Window Size: It shows the size of buffer in the receivers, so it will influence the communication procedure. So, change in window size reflects during transmitting.

4.1.2.2. Total number of TCP packets: This includes the total number of packets received at destination.

\section{PERFORMANCE EVALUATION AND SIMULATION RESULTS}

In this paper, an attempt was made to compare all the three protocols under the random way mobility scenario. The number of traffic sources was fixed at 10,20 and 30 , the maximum speed of the nodes was set to $20 \mathrm{~m} / \mathrm{s}$, the pause time was fixed and a fixed topology boundary of 500x400.

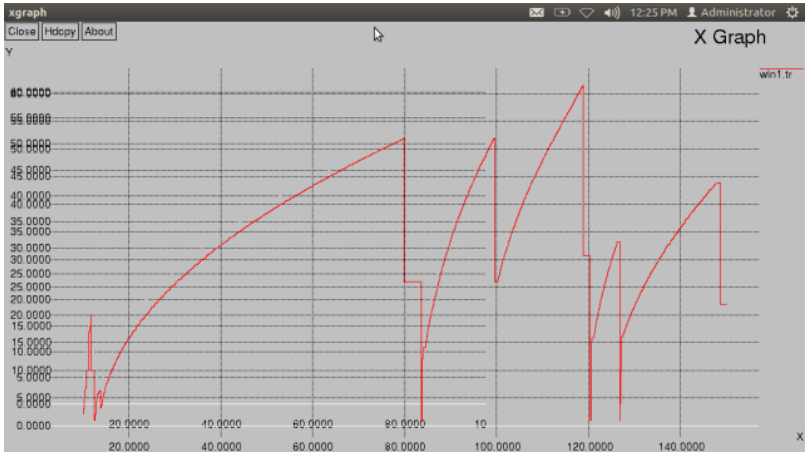

Figure 2: Window Size evolution of AODV (fixed 10 nodes)

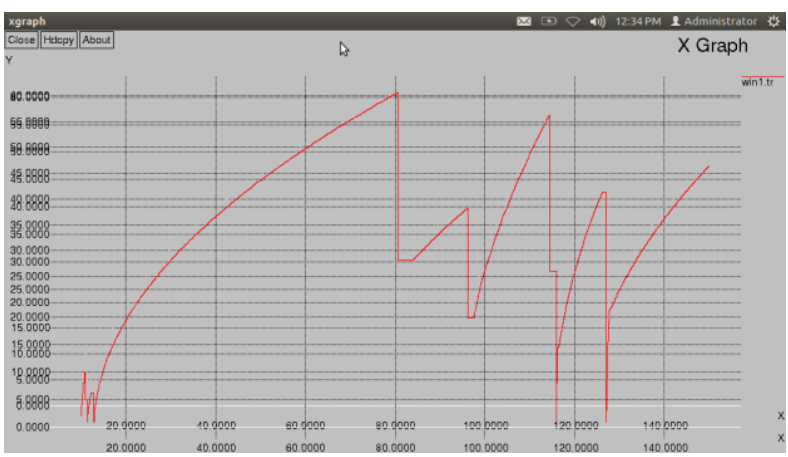

Figure 3: Window Size evolution of AODV (fixed 20 nodes)

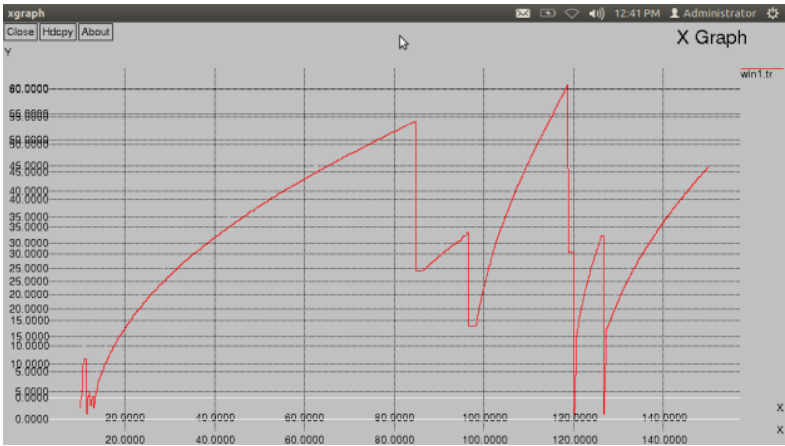

Figure 4: Window Size evolution of AODV (fixed 40 nodes)

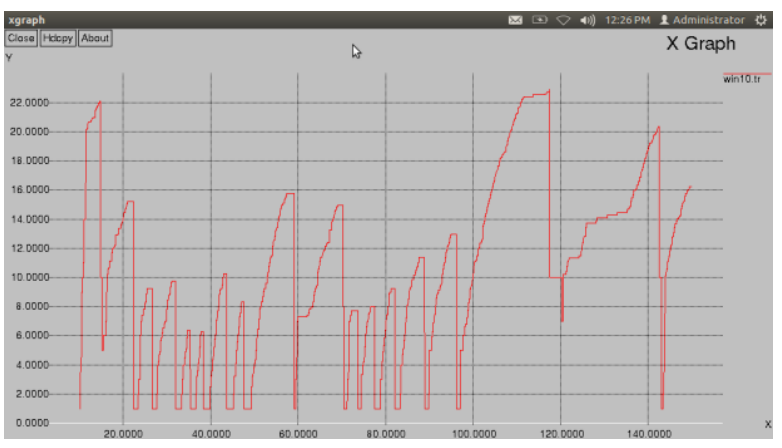

Figure 5: Window Size evolution of DSR (fixed 10 nodes) 


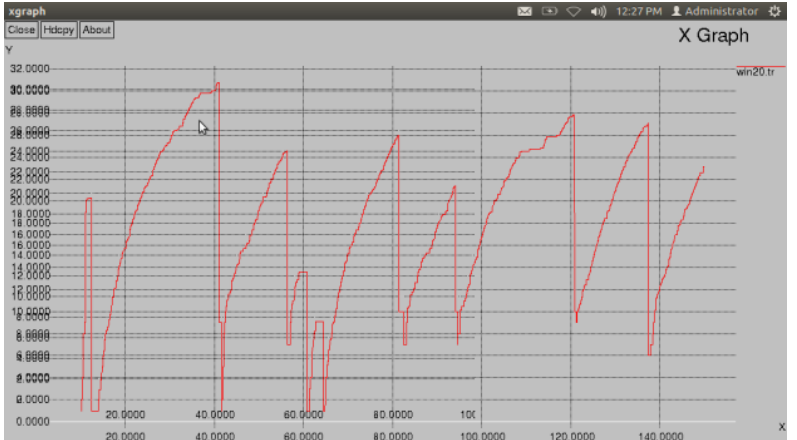

Figure 6: Window Size evolution of DSR (fixed 20 nodes)

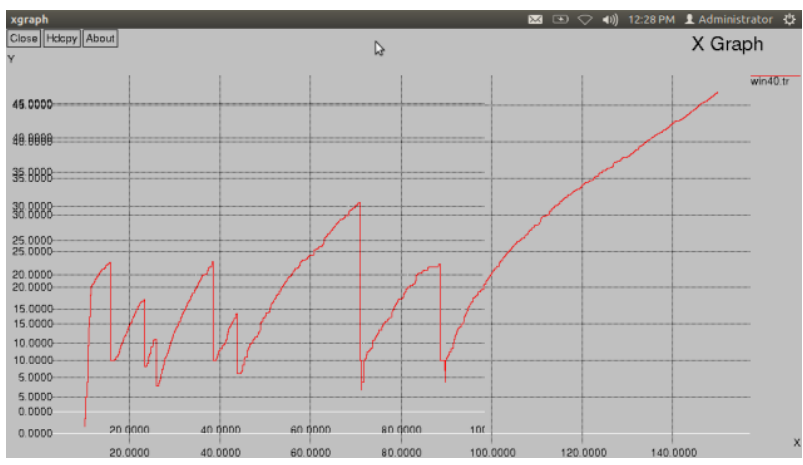

Figure 7: Window Size evolution of DSR (fixed 40 nodes)

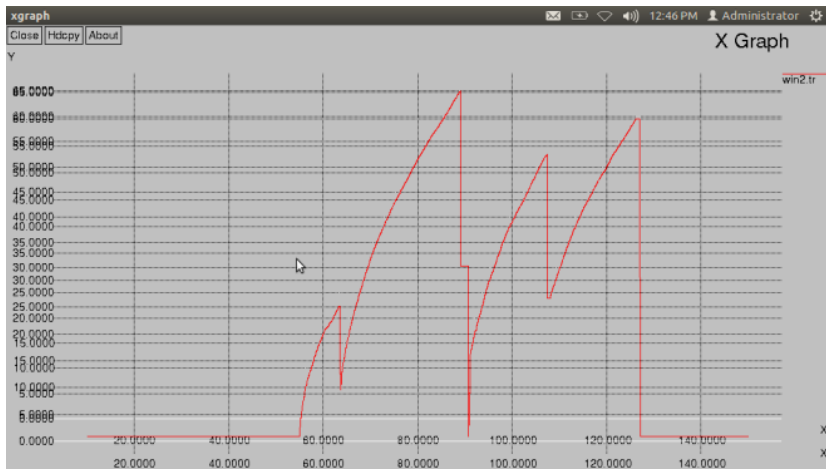

Figure 8: Window Size evolution of DSDV (fixed 10 nodes)

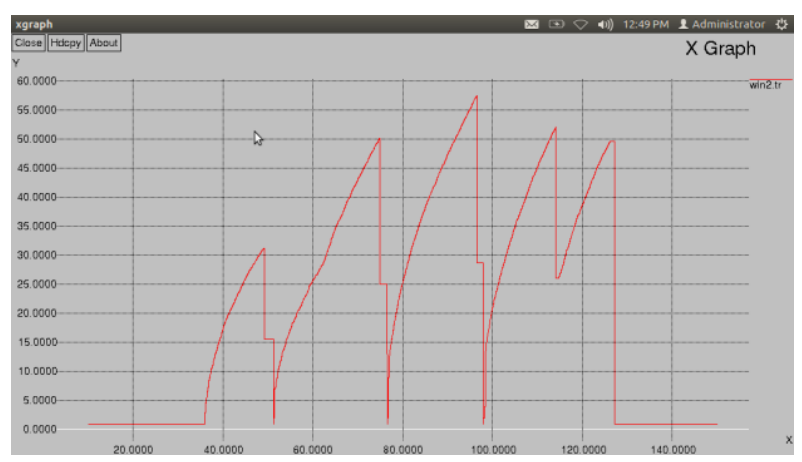

Figure 9: Window Size evolution of DSDV (fixed 20 nodes)

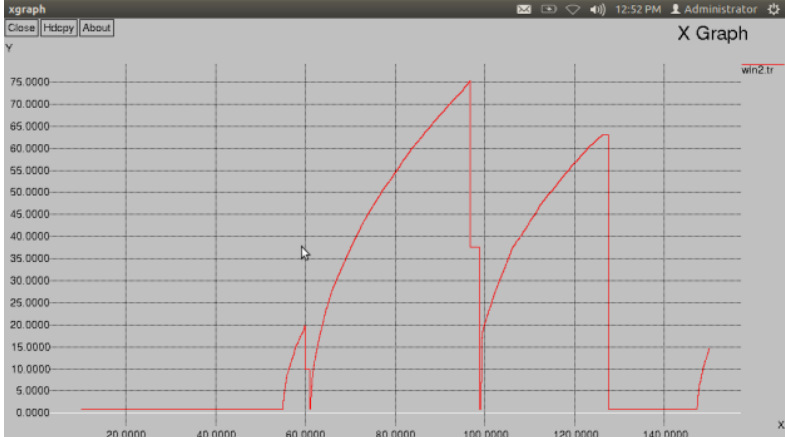

Figure 10: Window Size evolution of DSDV (fixed 40 nodes)

Results have shown that by using Random waypoint model for mobility, the On-demand protocols, DSR and AODV performed particularly well, the window size evolution on receiver side of DSR is very well. In AODV, evolution of window size on receiver size was similar with increase in number of nodes. But in DSDV, the system was not able to provide more phases for window size and connection was ended much earlier as nodes gets increasing. In other scenario, DSDV has transferred more packets than AODV even its window size evolution was ended much earlier. But DSR protocol has transferred most packets (TCP packets) as compared to other protocols with more number of nodes.

Table 1. Packet transferred in different routing protocols

\begin{tabular}{|c|c|}
\hline Routing Protocol & No of transferred packets \\
\hline AODV & 4658 \\
\hline DSR & 7144 \\
\hline DSDV & 5137 \\
\hline
\end{tabular}

\section{CONCLUSION}

Once the route has been established, the performance of AODV protocol shows better results throughout the simulation time except beginning and ending time. The Ondemand protocols, DSR and AODV performed particularly well with increased in number of loads, the window size evolution of AODV is very well. But in case of DSDV, it was not able to provide more phases and connection was ended much earlier. Therefore, DSDV has not shown good window size evolution as compared to On-demand protocols. High mobility results in link failures and overhead involved in updating all the nodes with the new routing information as in DSDV is much more than that involved AODV and DSR. DSR protocol has transferred most packets. So, Window size evolution of AODV protocol is very well and in other scenario DSR protocol most suits highly mobility systems with transfer maximum number of packets.

\section{REFERENCES}

[1] Tracy Camp, Jeff Boleng and Vanessa Davies, “ A survey of Mobility Models for Ad hoc Network Research", Wireless Communications and Mobile computing: A special issue on Ad hoc network Research, vol 2, No5, pp. 483-502, 2002. 
[2] Muhammad Abdulla, Robert Simon. 'Proceedings of the 41st Annual Simulation Symposium (anss-41 2008)', IEEE Computer Society, 2008.

[3] Fan Bai, Narayanan Sadagopan, Ahmed Helmy, "IMPORTANT: A framework to systematically analyze the Impact of Mobility on Performance of RouTing protocols for Adhoc NeTworks," in IEEE INFOCOM, 2003.

[4] P. Johansson, T. Larsson, N. Hedman, B.Mielczarek, and M. Degermark, "Scenario-based performance analysis of routing protocols for mobile Ad hoc networks," Proceedings of the 5th annual ACM/IEEE International Conference on Mobile Computing and Networking (MOBICOM '99), pp. 195-206, 1999.

[5] Yogesh Chaba, Yudhvir Singh and Manish Joon, "Simulation based Performance Analysis of On-Demand Routing Protocols in MANETs," in 2010 Second International Conference on Computer Modelling and Simulation, 2010.

[6] R. Groenevelt, P. Nain, and G. Koole. The message delay in mobile ad hoc networks. In Performance, 2005.

[7] Aravindhan Venkateswaran, Venkatesh Sarangan, Natarajan Gautam and Raj Acharya, 'Impact of mobility prediction on the temporal stability of MANET clustering algorithms', Proceedings of the 2nd ACM international workshop on Performance evaluation of wireless ad hoc, sensor, and ubiquitous networks, 2005

[8] Tracy Camp, Jeff Boleng, Vanessa Davies, "A Survey of Mobility Models for Ad Hoc Network Research," in Wireless Communications \& Mobile Computing (WCMC): Special issue on Mobile Ad Hoc Networking: Research, Trends and Applications, vol. 2, no. 5, pp. 483-502, 2002.

[9] Yasser Kamal Hassan, Mohamed Hashim Abd El-Aziz, and Ahmed Safwat Abd El-Radi, "Performance Evaluation of Mobility Speed over MANET Routing Protocols," in International Journal of Network Security, vol.11, No.3, pp. 101-111, Nov. 2010.

[10] J. Broch, D. Maltz, D. Johnson, Y. Hu, and J. Jetcheva. "Multi-Hop Wireless Ad Hoc Network Routing Protocols." ACM/IEEE International Conference on Mobile Computing and Networking (MOBICOM'98), pages 85-97, 1998.
[11] S. Lee, M. Gerla, and C. Chiang. "On-Demand Multicast Routing Protocol." IEEE Wireless Communications and Networking Conference (WCNC'99), 1999.

[12] E. Royer and C. Perkins. "Multicast Operation of the Ad hoc On-Demand Distance Vector Routing Protocol." ACM/IEEE International Conference onMobile Computing and Networking (MOBICOM'99), pages 207-218, 1999

[13] Nesargi and R. Prakash. "Distributed Wireless Channel Allocation in Networks with Mobile Base Stations." IEEE Conference on Computer Communications (INFOCOM '99), 1999.

[14] D.Sunitha,M. Chandrasekhar,"Measurement and Modeling of Mobile Ad-hoc Networks: It's Performance analysis using NS2", (IJAEST) International Journal of Advanced Engineering Sciences and Technologies Vol No. 9, Issue No. 2, 259 - 267, 2011.

[15] Mohd Izuan Mohd Saad, Zuriati Ahmad Zukarnain," Performance Analysis of Random-Based Mobility Models in MANET Routing Protocol." ISSN 1450-216X Vol.32 No.4 (2009), pp.444-454

[16] Santosh Kumar,S C Sharma, Bhupendra Suman,' Simulation Based Performance Analysis of Routing Protocols Using Random Waypoint Mobility Model in Mobile Ad Hoc Network," Global Journal of Computer Science and Technology,Volume 11 Issue 1 Version 1.0 February 2011.

[17] C. E. Perkins and P. Bhagwat,"Highly Dynamic Destination-Sequenced Distance-Vector Routing (DSDV) for Mobile Computers," Computer Communications Review, pp. 234-244, October 1994

[18] C. E. Perkins and E. M. Royer," Ad Hoc On Demand Distance Vector (AODV) Routing," Proceedings of 2nd IEEE Workshop on Mobile Computing Systems and Applications, February 1999.

[19] David B. Johnson, David A. Maltz, and Josh Broch,“ DSR: The Dynamic Source Routing Protocol for Multi Hop Wireless Ad Hoc Networks" in Ad Hoc Networking, edited by Charles E. Perkins, Chapter 5, pp. 139-172, Addison- Wesley, 2001 